\title{
Diffusion characteristics of dyeing in Polyester fiber garments referred to their mixtures with cotton and composition
}

Diffusion characteristics of dyeing in Polyester fiber garments referred to their mixtures with cotton and composition

\author{
Hugo Jiménez-Pacheco ${ }^{2}$, Gregorio Urbano Palma Figueroa ${ }^{1}$, Javier Alonso Villegas Aragon ${ }^{1}$, María del \\ Carmen Camiña Pacheco ${ }^{1}$, Antonio Erick Linares Flores Castro ${ }^{2}$, Lilia Mary Miranda Ramos ${ }^{1}$ \\ ${ }^{1}$ Escuela Profesional de Ingeniería Química, Universidad Nacional de San Agustín de Arequipa, Arequipa, Perú. \\ ${ }^{2}$ Vicerrectorado de Investigación, Universidad Catolica de Santa María, Arequipa, Perú
}

\section{INFORMACIÓN}

Historia del Artículo

Recepción: 18/06/2019

Revisión: 30/07/2019

Aceptación: 11/08/2019

Key Words

Diffusion, Gradient, Dyed, Hydrolytic.

\section{DOI}

https://doi.org/10.35286/veritas. v20i2.244

\begin{abstract}
Currently, textile dyeing companies determine their market according to the raw materials processed, which are based on $100 \%$ cotton fibers, these fibers are directed to an exclusive market and therefore these companies establish criteria of high quality standards in each of its processes, dyeing being one of the most demanding due to its fastness parameters and color specifications, these characteristics establish the competitiveness of expanding a product portfolio with the incursion of $100 \%$ fiber-based clothing polyester and with cotton blends. Some studies suggest the implementation of the dyeing process of these fibers, allowing the organization to have new production alternatives, dyeing processes for polyester and cotton are carried out with heat treatment between $60^{\circ} \mathrm{C}$ and $130^{\circ} \mathrm{C}$ respectively, with the use of dispersed and chemical dyes; as in the dyeing of Polyester-Cotton that are made in bathrooms established in each stage. Color and solidity tests are performed according to quality parameters, with light and wash fastness controls. Within these dyeing conditions it is essential to determine the thermal gradients to be applied, whose variable must be controlled to optimize the dyeing of polyester fibers and mixtures. The present study evaluates the pre-treatments and the temperature gradient behavior stage in the dyeing process of polyester fibers, taking into account the dyeing curve, in which only the temperature gradient varies. Mathis-ALT-B equipment is used for the dyeing process, for thermal gradient conditions of $135^{\circ} \mathrm{C}$ with gradients of $0.3-4.0^{\circ} \mathrm{C} / \mathrm{min}$, rotation speed $40-70$ RPM, and as colorants were used Novacron, Terail and Liandisperse for cotton and polyester fibers respectively. The results show that the use of dispersants and anti-breakage agents work to prevent failures and improve the corresponding colors, but a high gradient increases the migration of the dye and breaks in the fibers, but in low gradients $\left(2^{\circ} \mathrm{C} / \mathrm{min}\right)$ The quality of diffusion and dyeing is better and, therefore, prevents reprocessing in the dyeing stage.
\end{abstract}

\section{INTRODUCTION}

Currently the textile sector predominates, which becomes more competitive because companies must face the development of new methods that are used in the market, establishing differential conditions to be highly competitive (Gokhan Erkan, 2014), as in the case of fabric companies that establish resistance to light. Peru establishes a textile growth of 10 to $15 \%$ (Ana Marija Grancaric, 2013) and various companies in the area are involved in the export of garments based on polyester fibers and their mixtures with cotton; and within their processes they have the Dyeing, which establishes a substantial difference due to the pre-treatment of the main process, such as the solidity of the washing for discoloration and the presence of - $\mathrm{NO} 2$ in the chromoforms, responsible for increasing the affinity of the dye to cotton and the characteristics of solidity and resistance to rubbing of the fabric (Dan Gao, 2014). The implementation of this dyeing process in polyester fibers and their cotton blends is taken as a reference of quality standards (Sarkar A. K., 2015)

Correspondencia:

Hugo Jimenéz-Pacheco

hjimenezp@unsa.edu.pe taking into account aspects such as hue, color, standardized required tone and its depletion process, in a reduced time and somehow establish economic competitiveness (Arriaga A.M., 2013); the dyes present in the fibers present effective techniques in terms of enzymatic digestion methodologies to extract reactive dyes from the cotton fibers depending on the hydrolytic activity (Paulina Góra, 2019). Dyeing is established at $100 \%$ in cotton but not in its cotton and polyester blends where a depletion process is generated due to the volumes in each operation, this is due to the conditions of migration of the dye to the surface of the fiber, where the depletion rate of the dye is established according to the relative concentration, instead in the dyeing of the cotton fiber, its depletion establishes a better dyeing and improves the strength properties, its quality and the solids of the cationic dyeing compared to traditional dye dyeing (M. Ben Ticha, 2013). Therefore, it is established that the higher the concentration, the lower the depletion rate, this process determines the phase matching influenced by the dye concentrations such as the standardization of the dyeing process and the mass isolation of the dye material, which are predominant in its solids (Vankar P. S., 2019), these characteristics are established by the conditions of the thermal gradients and the specific characteristics of the fiber and its diffusion. Some of the aspects that determine the dyeing process are the influence of 
volumes to be treated, the length of the dye molecule and the applied power, establishing the viability of the technology and the correct dyeing process as well as its fine quality (V. Buscio, 2015), another aspect is the temperature, since at a higher temperature the adequate diffusion of the migration of the dye molecules to be dispersed in the established fibers and the enzymatic digestion that determines the effectiveness in extracting the dyes and whose activity is established hydrolytic is determined by the complete digestion of the fibers (Paulina Góra, 2019). According to studies, it is established the importance of the thermal variable with temperatures of 120 and $130{ }^{\circ} \mathrm{C}$ depending on the type of fiber or the structure of the modified molecular fiber and its pairing, so the temperature must be increased in reference to the dyeing kinetics . For low temperature dyeing conditions, conditions of agents that complement the pairing of parameters such as carriers or accelerators of molecules are identified, such as agents that establish their stability (E. Onder, 2015), anti-breakage agents such as dispersants for avoid breakage and its better pairing of its dye mixtures, establishing the properties of yarn and its structure and the dug treatment that lead to the highest quality of fibers (Burçin Ütebay, 2019), establishing the conditions of the cotton fiber and its mixtures with other fibers.

This research evaluates the different dyeing characteristics in polyester fibers, identifying the optimal gradients to achieve a quality product based on time and matching the dyeing kinetics.

\section{MATERIALS AND METHODS}

The dyeing process was carried out in $100 \%$ polyester fibers, and in 50/50\% polyester/cotton fiber blends, in $10 \mathrm{gr}$ samples and as dyes Novacron, Terail and Liandisperse were used. To carry out the dyeing, the Mathis-ALT-B equipment was used, with a thermal gradient condition of $135^{\circ} \mathrm{C}$ with temperature gradients of $0.3-4.0 \% \mathrm{~min}$, a rotation speed of 40 - 70 RPM, in 8 glasses of $200 \mathrm{ml}$ each, the FONG'SALLFIT 60 machine with HT technology was also used, with a fabric speed of 75-150 M / Minister, with a saturation steam heating medium of 6 to 8 bar overpressure. As for the coloring and dosage they are based on the standard colors and the visual approval LAB DIPS. Dryers were used for samples at temperatures of $130{ }^{\circ} \mathrm{C}$, a hydro extractor was used to squeeze the fiber at 50 PSI. Established characteristics of $100 \%$ polyester $150 / 34$ polyester fiber characteristics, redlight shade color referred to the characteristics of Terasil SD Yellow and Terasil SD Red. Auxiliary fibers for Merquiscour ESP dispersant polyester, Sodium Carbonate, Rucolin RSK anti-breaker, Acetic Acid, Caustic Soda, Hydrosulfite and Sapamine and for cotton fibers such as Invandina LU Moisturizer, Albaflow deaerator, Quelatex N sequestrant, MRN Hostapal soap, Caustic Soda, Acetic Acid, Cellusoft, Ladiquest 1094 sequestrant, Albafluid CD anti-seize, Amasalevel DL binding, Drimagen ER Liquid, Textile Salt, Sodium Carbonate and Sapamine, and its light fastness reference established to the NTP 231 005-1967 titration test method (INDECOPI, 2012) and the AATCC RA50 standards (American Chemical Association and Textile Colorists), whose tests of textile materials of all types and for dyes, finishes and treatments are established ICON-INSTITU T $\mathrm{GmbH}, 2008$.

\section{Lab tests}

In this stage a standardized dyeing curve process is performed, for the determination of the dyeing curves of PES and PES-CO according to dosage, auxiliaries, dyes and shades, in reference to the dyeing and washing time and the tests performed were considered according to the temperature gradient without taking into account the washing and softening processes of the fibers (Marielis C. Zambrano, 2019). The temperature gradients are established by an increase of up to $130{ }^{\circ} \mathrm{C}$ for the diffusion of the dye in the polyester fiber; and the decrease in temperature is given to avoid the breakage of the fibers, this occurs at $70^{\circ} \mathrm{C}$. For the pilot conditions dyeing curves were selected that have not exceeded $135^{\circ} \mathrm{C}$ as a technical specification in the machinery. The variables considered for environmental reasons are time, temperature, bath ratio, number of bathrooms, dyes and auxiliaries. And the temperature gradient variable, which can vary in the dyeing sub-process, considering that a higher value of the gradient means less time the importance of the temperature velocity intervenes in the coloring of the dye and therefore in the quality of washing fastness. In the case of polyester, 2 rinses are made for light tones, reductive washing continuously to dyeing for medium tones and reductive washing in two bathrooms for dark tones. The reason for the difference in washing is because at a higher hue, the non-fixed dye is of greater quantity and therefore requires a longer subprocess. In relation to the control tests, the dyeing time varies according to tones, being 45 minutes for dark tones and 30 minutes for light and medium tones; having an automatic control for being previously programmed in the dyeing machine.

\section{Experimental methodology}

The dyeing curve shows the dosage order, auxiliaries and dyes that match the time and the dyeing process. An average injection and wash time was considered; This curve is the same in all tests and the gradient varies only in the temperature rise. It was not considered the previous washing or the softening process of the fibers because it is not important for the purpose of this investigation. The temperature increase reaches $130^{\circ} \mathrm{C}$ and is shown in Fig. 1, this increase helps the dyeing process and the diffusion of the dye in the polyester fibers. And the temperature drop to $70^{\circ} \mathrm{C}$ prevents breakage in the fiber; However, a high temperature gradient can damage the fiber.

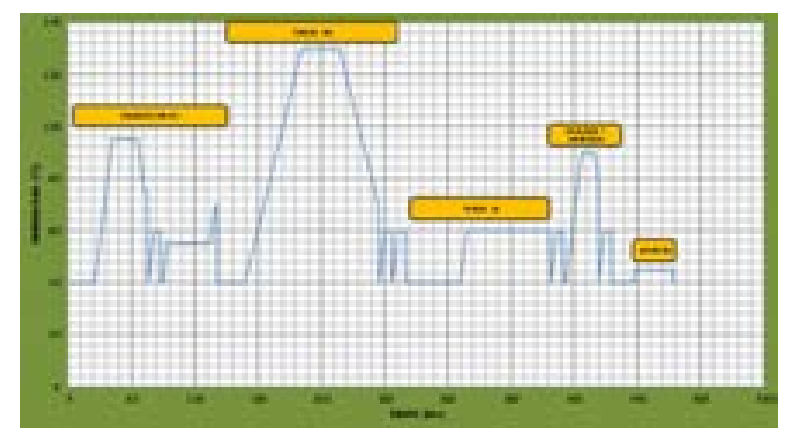

Figure 01: Characteristics represented for experimental tests. At the end of the dyeing process in the laboratory, samples are taken to the quality area to measure dye migration through the wash resistance test. 


\section{Wash fastness}

This test is carried out in accordance with the international standard ISO 105-C06 and is intended to specify methods to determine the color fastness of textiles of any nature and in all its transformation states, compared to the procedures of domestic and commercial washes Used for household items. There is a possibility that industrial or used items in hospitals may undergo special washing procedures that are more severe in some aspects. See Fig. 2. In the S (simple) type tests, degradation and discharge resulting from desorption and / or abrasive action correspond, with good approximation, to those that occur in a single domestic or commercial wash.

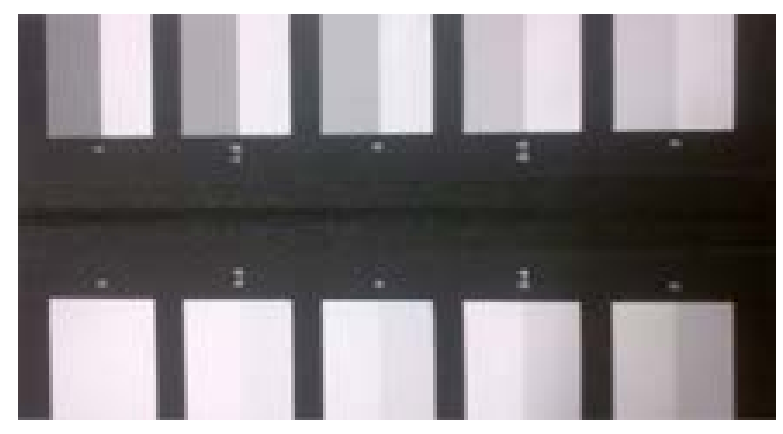

Figure 2: Grayscale - Washing fastness

\section{Dyeing process in polyester fibers}

The dyeing process in polyester fibers is selected and tested, and the result is a process adapted to the machinery, procedures and controls, and allows dyeing with high light and washing fastness to be obtained. The dyeing process classifies different colors (light, medium and dark), which are tested in the laboratory with $10 \mathrm{gr}$ samples and in the plant with $50 \mathrm{~kg}$ samples. The dyeing curve selection was established according to the research found on the dyeing of the PES and PES-CO fibers.

\section{RESULTS AND DISCUSSION}

Experimental tests determine aspects of granulation and show a poor correspondence of the dye in the entire fiber, a regular fastness to washing, a dye speed and a form of fixation (Joaquina Albán-Castillo, 2018), because the high gradient prevents the gradual diffusion of the fiber and the dye in the fiber. The dye has a better resistance to washing with a gradient of $2{ }^{\circ} \mathrm{C} / \mathrm{min}$ and this results in a better dyeing quality and a better time-saving ratio because it prevents redoing the dyeing process.

The initial dyeing speed doubles in the region between $100{ }^{\circ} \mathrm{C}$ and $140{ }^{\circ} \mathrm{C}$ when dyeing polyester fibers so it is necessary that the bath current is sufficiently intense and uniform for each circuit to pass through the fabric at the same temperature and the same concentration. The bath should be fast enough in relation to the rate of dye increase (Jimenez P, 2016). The faster it heats up, the softer the dye feed and bath circulation should be.

Light fastness corresponds to NTP 231.005: 1967 (INDECOPI, 2012), see Fig. 3. The test methodology was established to assess the color fastness of textile materials, in all their degrees of transformation, to the action of daylight, and there was a validation of the AATCC 16 RA50
(American Association of Textile Chemists and Colorists), which establishes the general principles and procedures that are currently in use to determine the color fastness in light of the textile materials.

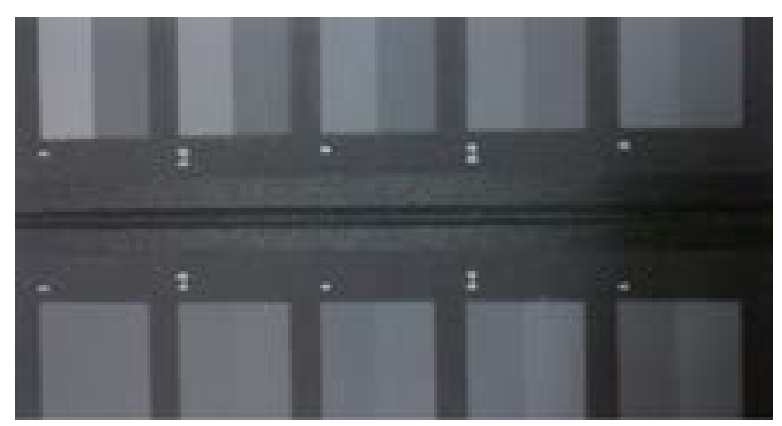

Figure 3: Characteristics of color fastness to light

These described options are applicable to textile materials of all types and for dyes, finishes and treatments applied to textile materials (Icon-Institut $\mathrm{GmbH}, 2008$ ).

\section{CONCLUSIONS}

Graduating less time means less dyeing time, and this does not meet quality standards. And the dispersants and auxiliaries used allow the correct homogenization.

The dyeing of polyester fibers in a gradient of $2{ }^{\circ} \mathrm{C} / \mathrm{min}$ allows a complete coincidence of the dye in the fiber, which allows a high diffusion that greatly reduces the migration of the dye and prevents the fiber from breaking.

Quality tests are mainly based on washing fastness and light fastness, although there are other tests that are carried out, stipulated under different and exclusive procedures for textile companies. While the technical standards establish quality and safety levels and are an optimal means to facilitate transparency in the market, such as sustainability to be achieved according to the harmful effects on the preservation of the environment (FA Esteve-Turrillas, 2017); and fundamental conditions to compete (INDECOPI, 2012); considering the quality policy of various textile companies that establish their exclusive market.

\section{ACKNOWLEDGMENT}

A cordial thanks is extended to the Institute of Research and Innovation in Renewable En-ergies and Environment - Innovergy of the Catholic University of Santa María, to the Pro-fessional School of Chemical Engineering of the National University of San Agustín and to the Company Franky \& Ricky SA, especially to Mg. Abraham Carrasco.

\section{REFERENCES}

1. Ana Marija Grancaric, I. J. (2013). Bioplastics in Textiles. Bioplastics, Society of Plastics and Rubber Engineers, 1/9-14.

2. Arriaga A.M., L. T.-N. (2013). Chemical composition and antioxidant activity of Indigofera suffruticosa. Chemistry of Natural Compounds, 49/150-151.

3. Burçin Ütebay, P. Ç. (2019). Effects of cotton textile waste properties on recycled fibre quality. Journal of Cleaner Production 222 (2019) 29e35, 222/29-35.

4. Dan Gao, H.-s. C.-t.-f.-x. (2014). Synthesis of reactive 
disperse dyes containing halogenated acetamide group for dyeing cotton fabric in supercritical carbon dioxide. J. of Supercritical Fluids, 86/108-114.

5. E. Onder, N. S. (2015). Thermal regulation fi nishes for textiles. Functional Finishes for Textiles, 17-98.

6. F.A. Esteve-Turrillas, M. d. (2017). Environmental impact of Recover cotton in textile industry. Resources, Conservation and Recycling, 116/107-115.

7. Gokhan Erkan, K. S. (2014). Dyeing of white and indigo dyed cotton fabrics with Mimosa tenuiflora extract. Journal of Saudi Chemical Society, 18/139-148.

8. Jimenez P, H. D. (2016). Process Optimization in the Dyeing of Polyester Fibers Through the Control Variables Temperature Gradient. ARPN Journal of Engineering and Applied Sciences, 1819-6608.

9. Joaquina Albán-Castillo, G. E. (2018). Color in the Memory: Plant Dyes Used in the Traditions of Andean and Amazonian Peruvian Communities. Ecología Aplicada, 1726-2216.

10. M. Ben Ticha, N. M. (2013). A promising route to dye cotton by indigo with an ecological exhaustion process: A dyeing process optimization based on a response surface methodology. Industrial Crops and Products, 46/350-358.

11. Marielis C. Zambrano, J. J. (2019). Microfibers generated from the laundering of cotton, rayon and polyester based fabrics and their aquatic biodegradation. Marine Pollution Bulletin 142 (2019) 394-407, 142 / 394-407.

12. Paulina Góra, J. W.-G. (2019). Enzymatic extraction of dyes for differentiation of red cotton fibres by TLC coupled with VSC. Science \& Justice 59 (2019) 425432, 59/425-432.

13. Sarkar, A. (2015). Dyeing technologies for denim garments. Dyeing technologies for denim garments, 272-285.

14. Sarkar, A. K. (2015). Dyeing technologies for denim garments. Dyeing technologies for denim garments, 271-285.

15. V. Buscio, M. C.-B. (2015). Sustainable dyeing of denim using indigo dye recovered with polyvinylidene difluoride ultrafiltration membranes. Journal of Cleaner Production 91 (2015) 201e207, 91/201-207.

16. Vankar, P. S. (2019). Dyeing Application of Newer Natural Dyes on Cotton Silk and Wool With Fastness Properties CIE Lab Values, and Shade Card. New Trends in Natural Dyes for Textiles, 159-282. 